\title{
Cross-Sectoral Mobility Funding and the Challenge of Immersion: The Case of SSH
}

\author{
Tomas Hellström ${ }^{1}$ (D) Christina Hellström ${ }^{2}$
}

Published online: 5 March 2020

(C) The Author(s) 2020

\begin{abstract}
Project funding rarely demands much change on behalf of the recipient. In contrast, cross-sectoral mobility funding requires recipients to change their environment and often some aspects of their research. There is a need to understand the impact on the researchers' experiences as knowledge producers within such programs, as part of the broader potential and significance of cross-sectoral mobility funding. This study draws on interviews with participants of the Swedish 'Flexit' program in order to develop a framework for assessing the dynamics and efficacy of such funding instruments. To do this, we develop a framework for understanding their cognitive effects; especially their 'immersive' potential, i.e., their ability to naturally involve the participant in their new setting. It proposes two dimensions along which such an assessment can take place: how the instrument challenges participants' knowledge production practices, and the level of immersion that participants are subject to as part of the program.
\end{abstract}

Keywords Research funding · Funding instrument · Experience $\cdot$ Immersive . Impact $\cdot$ Methodology $\cdot \mathrm{SSH}$

\section{Introduction}

Inquiry into the functions and effects of policy instruments has been part of the policy studies agenda for some time now (e.g., Salamon 1981; Hood 1986; John 2013). For the field of science policy studies, much of this research takes on an evaluative

Tomas Hellström

tomas.hellstrom@fek.lu.se

1 School of Economics and Management, Lund University, 22100 Lund, Sweden

2 Department of Psychology, Göteborg University, Göteborg, Sweden 
character, and focuses on various forms of additionality from types of policy programs for Research and Development (R\&D), including that of behavioral additionality, i.e., changes in practices among the targets of a program (Luukkonen 2000; Clarysse et al. 2009). Rip's $(1981,1997)$ socio-cognitive approach to science policy is one instantiation of this. Rip contends that science policy needs to build on an understanding of how: (i) the social and cognitive norms of science interrelate, and (ii) how the social and cognitive norms of politics and administration themselves relate to scientific norms. For this type of analysis to work, however, one needs to build on a solid understanding of how instrument characteristics mediate and affect specific types of researcher actions, which includes the question of how researchers perceive or experience being subject to the influence of such instruments. Gläser and Laudel (2016) make a related argument when they assert that a research policy can have an impact on knowledge production only if it affects what the researcher does. For Gläser and Laudel, the researcher is the necessary passage point for any instrument's influence on knowledge production. The above may be read as an argument for further investigation of how funding instruments affect researchers' views of themselves as knowledge producers in the context of a specific instrument.

The paper presents a study of the experiential effects on researchers of a research funding instrument. There is a growing literature on the differential impacts of research funding and evaluation on researcher behavior and output. The main insights from this literature revolve around the relation between bibliometric valuations and problem choice, publishing behavior and 'evasive tactics' (Whitley 2007; Laudel and Gläser 2014; de Rijcke et al. 2016). Others have investigated the organizational effects, e.g., collaborative practices, from new forms of 'collectivist' funding schemes like Centers of Excellence (CoEs) (Bishop et al. 2014; Brorstad Borlaug 2016; Hellström 2018). A recent special issue in Minerva on 'changing funding arrangements and the production of scientific knowledge' deals with some of the general issues addressed above, specifically the impact of funding instruments on research practices and content (Gläser and Serrano Velarde 2018). Here, Gläser and Serrano Velarde (2018) note that research funding schemes are increasingly used to "influence the conduct and content of research through the modification of funding arrangements [for example] by incorporating [...] public policy goals in funding programmes" (p. 2).

The present paper contributes to and extends this body of research by outlining and applying a theoretical framework for studying how participation in an immersive' funding context influences the researcher's experience as knowledge producer. Immersive funding instruments are on the opposite side of the spectrum from traditional project funding because they require the recipient to engage in specific types of behaviors/environments during the funding period. A moderately immersive instrument would be $\mathrm{CoE}$ funding, which requires the researchers to be part of a specific thematic research organization (Hellström 2018), and a highly immersive instrument would be the type of research internship described in the present study. The notion of immersion as a cognitive concept has been thoroughly addressed in the Human-Computer Interaction (HCI) and computer gaming literature, where it is usually taken to denote the experience of spatial presence and flow (involvement in action) in a mediated world (Weibel et al. 2010). The literature suggests 
that immersion in a new 'world' or context depends on motivation of the actor and some other cognitive characteristics, e.g., 'immersive tendency' (Witmer and Singer 1998), and that immersion under such circumstances stimulates enjoyment and performance (Weibel et al. 2010). An instrument can be referred to as immersive when its intended modus operandi is to reposition an actor into a new setting, thereby affording the actor new insight and experience, and stimulating new actions, in this case in terms of doing research. Such an instrument may not succeed in this, but when it does it is due to it having conferred an experience of immersion upon the participating actor. The notion of an immersive instrument therefore includes the idea of a new environment, and an experience of immersion, the latter being always contingent on circumstances. In the following, we will use the notion of immersion in this dual sense. The aim of this paper is to outline some elements/expectations on such instruments using an an empirical qualitative study from the social sciences and humanities ( $\mathrm{SSH}$ ), and further to suggest conditions under which such an instrument can succeed.

The study focuses on 'Flexit,' a funding instrument developed by the Swedish Foundation for Humanities and Social Sciences (RJ) that aims to introduce early to mid-career SSH scholars into firms, governmental, and non-governmental organizations. This is achieved through an internship program where researchers spend three years full-time in the host organization, during which they are expected to develop a research project relevant to that organization. They also spend some of their time (25\%) working as ordinary employees, taking regular tasks. This makes it a highly immersive instrument in terms of researcher experience.

As far as funding instruments are concerned, the case is unusual due to its focus on SSH. Much of the SSH field is what Whitley (2003) describes as 'differentiated pluralist' in that researchers are subject to high levels of intellectual pluralism and flexibility, and weak competition for national and international reputation. This is a condition they share with engineering fields, but without the traditional practical problem-solving leaning of these disciplines. In this way SSH could be said to represent a 'critical case' for this type of immersive, practice-oriented instrument. In what follows we will suggest a framework for analyzing the experiential influences of such an instrument on participating researchers, which builds on an understanding of the cognitive dimensions of policy instruments, and an interpretation of these in terms of their phenomenological, or experiential 'life-world' implications. Second, the case will be described along with the method employed in data collection and analysis. Third, an analysis of experiential impacts will be outlined, illustrated with quotes from the researchers, and finally a discussion focusing on how such an analysis can aid in understanding the affordances of research funding instruments, specifically for immersive instruments, but also in general. Here, in the final part of the paper we will outline an approach to analyzing the experiential impacts of immersive funding instruments, and suggest ways to promote their usefulness to research. Specifically we will attempt to address the questions: To what extent can an immersive instrument become a natural part of the researcher's everyday experience or, as we will describe it below, 'fade into the researcher's background'? Under what conditions does the instrument offer an immersive experience in this way, rather than one where the researcher resists immersion or experiences being 
resisted by the environment? And to what extent does the instrument challenge the researcher, in terms of helping him/her develop in new directions?

\section{Experiencing Instruments: Mediation and Immersion}

The idea that policy instruments have cognitive effects and that they depend on cognitive dispositions is not new (for an overview, see Hellström and Jacob 2017). Lascoumes and Le Gales (2007), for example, define a public policy instrument in the following way: "...a device that is both technical and social, that organizes specific social relations between the state and those it is addressed to, according to the representations and meanings it carries" (p. 4). In this paper, we are particularly interested in the experiential aspects of instruments, i.e., how they may affect the subject's environment and their involvement in this environment in various ways, i.e., their life-world experience. The point of departure here is that the policy instrument is a technology not just for the policymaker, but also for the user. While in the traditional conception the policymaker is the subject, who uses the policy instrument or tool to dispense steering towards an object/user, in this analysis the subject/object relation is reversed so that the user is the subject who 'uses' the instrument at various levels of involvement. In approaching the problem of instrument effects from this perspective, an important question is to what extent the experience of being involved with a policy instrument represents a friction, that is, whether the instrument/life-world relation is a resistant and problematic one, or whether the relationship is smooth, non-disruptive and integrative (Ihde 2004).

This question is to some extent already addressed in the policy literature, however indirectly. For example, Linder and Peters (1989) discuss design criteria for instruments in terms of their adaptability across users and their level of intrusiveness. Linder and Peters assume that an instrument that 'fits' into a user's specific circumstances and expectations is more likely to work than one that does not. In his now classic statement on policy instruments, Hood (1986) similarly suggests that instrument choice should depend on the character of the targeted group and that policymakers should prefer instruments with low complexity, low visibility, and low intrusiveness. Building to a certain extent on the previous authors, Salamon and Elliott (2002) propose that one categorizes policy tools according to four dimensions: directedness, visibility, coerciveness and automaticity. All these dimensions are in one way or the other related to the immersive potential of a policy instrument, i.e., how adapted, voluntary and discernable it is to the target. This is essentially tantamount to saying that policy instruments qua 'life-world presences' may be more or less visible/transparent to the user, immersive and thus more or less easy to integrate as part of the subject's previous dispositions. An instrument can be subject to resistance or acceptance, and cause various amounts of frustration/concern in the subject.

One corresponding notion in the phenomenology of technology is that technology mediates between the experiential subject and the experienced object world. Technology, or a policy instrument in this case, is taken into experience by the way in which one perceives through technology, and how one adapts perceptual and body senses to this experience, for example, through social expectations and concomitant 
Fig. 1 Technology mediates world perception. (Adapted from Ihde 1990)

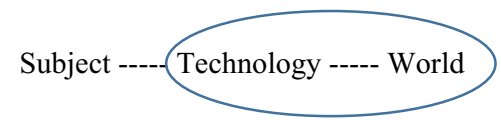

or

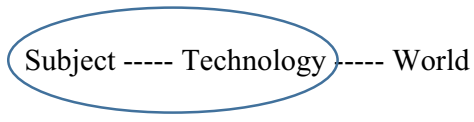

behavior (Ihde 1990, 2004). This tripartite relationship is illustrated in Fig. 1. Following Ihde (1990) one may imagine an integrated or immersive subject/technology relationship that together comes to represent the actor experience of the world, or reversed a more frictional subject experience of technology and world as separate from the subject, i.e., a 'technologyworld' that stands apart from the experiencing subject. In this version technology is experienced as something outside of the subject that has to be 'dealt with.' When an actor is immersed in the technology s/he 'sees' through the technology. To use Merleau-Ponty's famous example "the blind man's stick has ceased to be an object for him and is no longer perceived for itself; its point has become an area of sensitivity, extending the scope and active radius of touch, and providing a parallel to sight" (Merleau-Ponty 1962: 167).

Lascoumes and Le Gales (2007) have expressed this enabling and immersive character of policy instruments by referring to them as institutions, in the sense that they create 'capacities for action' in various ways, e.g., 'structures of opportunity' in the way resources are identified and used, and by whom. The idea of instruments as institutions is closely tied to the above notion of immersion and mediation of technology/instruments. The phenomenology of technology recognizes that an efficacious technology must be 'transparent,' in the sense that it can be 'seen through' by the subject. This is a metaphor for the technology fading into the background and becoming an intuitive part of the subject's life-world. As a corollary to the idea of structures of opportunity, Radaelli and Meuwese (2010) have noted that instruments also facilitate learning by generating such structures. As we shall see below, this is a quality that may be designed into or discovered in policy instruments. According to this way of thinking, the key is that instruments must reduce the subject-instrument relation to a 'face-to-face' or 'close-in-sight' level, so that the mediated experience is close to the actor's actual bodily position and perception, and in that sense similar to a non-mediated situation (Ihde 1990). The idea of having access to the outside world primarily through active involvement with it is owed to Heidegger (1962) and the notion of Dasein ('being in the world'); a state in which we are always acting unreflectively with the objects that are 'ready-to-hand' to use Heidegger's phraseology. Another way of saying this is that instruments should have a natural relation to the subject, i.e., be part of the way they normally do things or think about themselves.

There are several ways in which this ideal subject-instrument-world relation can be either thwarted or stimulated. For one, certain problems naturally 'promote' certain instruments. One may, therefore, speak of an 'instrument-problem fit' that may be 
more or less appropriate (Elmore 1987). In addition, a policy program usually needs several instruments, and one instrument may be composed of a number of supporting sub-instruments where some features are subsidiary to other more central ones. Some of these may be 'leading' and some 'following,' and this ensemble of influences must, of course, cohere vis-à-vis the subject (Elmore 1987). In this way, one may identify conflicting impulses/signals deriving from one or several instruments that impact on the subject, or what Kassim and Le Galés (2010) refer to as conflicting levels of instruments. Because of such instrument complexity and the possible intersection of several instruments impinging on the actor, the subject experience of acting under the conditions of an instrument may be 'polymorphous' or ambiguous. The instrument may reinforce the subject's abilities and goals i.e., one can do more in the 'instrument world,' but at the cost of a more complex and possibly conflictual experience (Ihde 1990).

Apart from sending conflicting messages, an instrument may also send one message but in a way that meets resistance. Lowi (1972) categorized policies according to their coerciveness, and Doern (1981) following Lowi suggested a one-dimensional criteria for instruments, namely, that of 'degree of legitimate coercion.' He argued that liberaldemocratic societies ought always to choose the least coercive instruments possible, and to move up the coerciveness scale only when necessary. Funding instruments have traditionally been of a non-coercive and highly voluntarist nature (goes along with the notion of 'academic freedom') and any funding instrument is likely to fail if it is experienced as illegitimately coercive. This ties into the relationship between instrument goals (which may be more or less explicated) and effects (many of which are usually unknown in advance), on the one hand, and the goals and sentiments of the subject of the other. When instruments potentially affect the enactment of professional norms, as is the case for many funding instruments, they must be able to act in consonance with such norms or to legitimately adjust them (see Doern 1981 above). One may say that instruments depend on/appeal to goodwill or the 'sharing of norms,' or on the sharing of interest to act (John 2013).

In summary, and following Ihde (1990) and others, there must be compatibility between the subject/user and technology/context for an instrument to be efficacious. From a phenomenological perspective, this is more than a technical design issue. An instrument that fits the use must be 'available' and beneficial to the user, e.g., ensure feedback and reinforcement of sentiments, yet withdraw and become invisible in use (immersion). According to this way of thinking, if the instrument is good, it will 'withdraw' and become maximally transparent and embodied by the subject, and thus come to constitute the subject's world experience without itself being visible. However, often such instrument embodiment must be learned or adapted to the specific local experience of the subject. The ease with which this is done might be a sign of the quality of the instrument. In the empirical section below we will see several examples of such immersion, but also of resistance to instrument immersion. 


\section{Case Background and Method}

\section{The Instrument: Case Background and Participants}

The empirical study focuses on an immersive funding program aimed at facilitating exchange between university (SSH) researchers, and various 'non-academic' environments through an internship approach. To this end the 'Flexit' program aims to build bridges between SSH research and organizations outside the academic sphere (private, public and third sector) in order to demonstrate these actors' value to each other. According to program goal statements, this may lead to alternative career paths for academics and practitioners and, in the long run, possibly affect traditional notions of professional merit, inside and outside of the academic sphere. This is done by offering internships in non-academic settings for early to mid-career SSH researchers. Researchers apply to be part of the program with a CV and a research plan, and if successful spend three years full-time with the external organization (a second round of researchers spend two years in the organization and the last year in their home department). During this time, they are expected to conduct a research project connected to the organization, and they have a supervisor there (RJ 2018). Twice a year the Flexit-researchers meet with fellow program participants for a oneday workshop to exchange experiences. Apart from that, they are left to their own devices, to work in this dual role as researcher/practitioner under the supervision of their respective organizational mentor. The participants reported a number of motives for applying to the program, the most salient of which were (i) the interest in 'doing something new,' (ii) to try out one's research in an applied context, or (iii) simply to be able to maintain a university connection after the $\mathrm{PhD}$. Common to all participants, however, was a general interest in trying out working in an applied setting. Some of these motives are revealed in the interview quotes, but apart from that no systematic influence of motives on the participation experience could be discerned from these interviews. Since 2010, 32 internships have been initiated in disciplines ranging from philosophy to business administration. Of these the present study selected 18 participants from a representative range in terms of disciplinary affiliation and host organizations. Type of host organization covered private, public and NGOs, and included from engineering to publishing houses on the private side of the spectrum.

\section{The Methodological Approach}

The empirical study employed an approach based on interpretative phenomenological analysis (IPA). The typical aim of this method is to explore in detail how a person or group engages in and experiences an activity in the sense of assigning meaning to this activity. The approach is phenomenological in that it emphasizes the meanings of particular events, states and experiences as these hold for the participants (Smith and Osborn 2003). The researcher works out interpretations to a large extent already present in the expressions of the participants, i.e., the subjects 
own elaborations and sense-making (Smith 1996). Because of the focus on meaning structure and personal experience, the standard measures of representativeness of sampling are replaced in phenomenological analysis with an attention to detail of expression, and a probing attitude to the interview itself and its resulting material. In addition to this, the phenomenological approach usually focuses on creating a homogeneous sample about the phenomenon under study (Smith and Osborn 2003). The richness of the data generated in this type of study, as well as the emphasis on description and exemplification of case-specific instantiations, reduces the need for many participants but increases the demand on the researcher's attention to specific meaning carrying expressions of the participants.

\section{Procedure}

In-depth interviews (18) were conducted in an informal, conversational manner, on an individual basis, in a quiet room often in the participants' workplace. The interviews lasted for about 1-2 hours and were recorded. The interviews focused on the participants' experiences of the Flexit program, and the relation between the circumstances of the program, participants' immediate experience of the context/presence in the host organization, and their relationship to specific conditions that the program established for them. Examples of questions were 'how did you experience the Flexit programme?'; 'what were your experiences in the host organization?'; 'how did these experiences affect you and your goals?' The questions aimed to facilitate the participant telling his/her own story, not to check the investigator's preconception of the phenomenon studied (Giorgi and Giorgi 2003). Any issues that illuminated the processes and experiences of the Flexit program, which arose in the conversation, were further probed through explicit non-directive questions.

\section{Data Analysis}

Interviews were transcribed verbatim into protocols. The interview protocols were read in order to establish significant passages, interpretative flexibility and direction of the general narrative. The individual protocols were then re-read line by line and broken down into discrete parts, or meaning units, i.e., where a visible change of meaning could be discerned (Giorgi and Giorgi 2003). These units were clustered into categories, each of which captured some specific homogeneous quality of what was said by the participants. Categories were ordered according to the way participants placed expressions in a valuational narrative, i.e., whether they regarded a particular experiential aspect as positive or negative to the general Flexit involvement. This was an ordering pattern that emerged through the analysis rather than one imposed on the material.

The following section presents these categories with illustrative quotes from the interviews. These quotes are analyzed and interpreted as they are presented. This gives an interpretative perspective on the interview quotes as well as providing an analytical context for the discussion and conclusions. 


\section{Results}

The interviews revealed a number of experiential dimensions, and two turned out to be especially salient in the material as a whole. The first is a negative dimension which involves the experience of uncertainty regarding the new situation, and of friction between oneself and one's new setting. The second is positive and describes a learning experience, where the actor adapts and develops given the new circumstances. This experience contained additional elements of innovation or taking a leap forward compared to one's previous abilities and conditions. Below we will use the subcategories organizing the material to suggest how uncertainty and friction and learning and development are constituted. From what can be gleaned, expectations, problems and research agenda hang together and are all subject to uncertainty of the new situation, while culture and role identity correspond to each other, and are similarly subject to ambiguity and friction. In the case of learning and development we see how the new culture provides opportunities for developing one's identity as a researcher and subsequently to engage in new types of research processes. ${ }^{1}$ In what follows we will explain and illustrate the two dimensions using quotes from the interviews.

\section{Uncertainty and Friction}

This dimension describes the researcher's sense of complexity, uncertainty and concomitant experience of friction, about his/her presence in the organization. One point of uncertainty regarded expectations, from both funder and host organization, as to what the researcher was supposed to do. As one participant expressed it about the funder:

I didn't have, you know a discussion concerning what was expected from their point of view, we never had that...

There were also difficulties in understanding the host's expectations of the recipient, e.g.:

I don't think I understood what the organization was really asking for...I think they had a hard time expressing what they wanted...I'm not sure they knew themselves exactly what they wanted...

The difficulty that researchers reported having about communicating and understanding expectations is, in several ways, related to uncertainty about the problems that the researcher should address during his/her stay. Experience of uncertainty about the practitioner's problem starts already before the internship has begun, e.g.:

There is very little description in the call. There is this gap, and we need to work with this...I didn't get any more information about what they wanted.

\footnotetext{
1 We wish to thank an anonymous reviewer for pointing out that these subcategories may be applied for understanding impact from other types of research funding instruments.
} 
Even though the researcher has prepared a case problem and cleared it with the funder and the host, once they arrive in the organization uncertainty is unavoidable due to the practitioners' closer connection to the (practical) research object:

When I came to the company...I had to revise everything...I mean their understanding was much more... I had to adjust... my project as well. That was very difficult...they were running much, much faster.

Formulating a research topic that could work for both academic and the host organization also posed obstacles. These were due to a number of non-explicated expectations where researcher identity played a role, e.g.:

Whether it's because I was quite junior or because of the context ...being a different kind of researcher but at the same time producing research that would be acceptable in a university department... what kind of research should I produce ... what is the field, what is the discourse ... it really took some time to get into that role and ... to actually handle these people.

What they said was that "we need to find ways for you to work that ...makes sense for us.'

Conflicts regarding the formulation of problems and topics illustrate difficulties in combining the academic's research agenda with the organization's agenda.

I already had my research planned and everything... the difficult part of my project was to cooperate with them and stick to my own plan and my own interests... It's been difficult to give priority to my own work.

This could sometimes become quite a matter of identity:

When am I their colleague? When am I the researcher? ... How [do] they understand what I'm saying?... what will I write? [what I write to] help them forward and the things I write in the academic articles may not be the same thing...

As can be read from the above quotes, the 'intellectual friction' between researcher and organization sometimes had important social implications. This friction was amplified by the fact that the researcher was operating in relative isolation from other researchers, physically and intellectually, e.g.:

So the main problem is you're alone as a researcher ...you're in an organization with no other researchers ...you don't have a project that involves other researchers ...And I think that's very stressful... and it's also difficult to collaborate with other researchers because they are tied to what they do. To the research money they have.

This quote shows how researcher identity, problem choice and cultural differences coincide to produce an experience of uncertainty in the researcher. Culture was an important source of uncertainty among participants because the stable points of reference they were used to were often not shared by the practitioners: 
...but my experience from [firm], and I would imagine that quite a lot of researchers in the Flexit program experience the same thing, is that you don't have... a shared ... point of reference concerning how you approach different kinds of ...problems or challenges.

This expresses a form of cultural uncertainty - lack of shared reference points - where the implications are difficult to assess outside of daily experience since it relates to how issues are dealt with continuously. It represents a very tangible form of friction for the participant. In what follows we will deal with friction more specifically since this turned out to be a recurring aspect of the researcher experience in the program. The researchers' accounts contained many examples of what may be described as instances of a clash between academic and practitioner work culture. This clash is in some respects related to culturally predicated expectations, e.g.:

I mean when you come from academia, you are used to, 'here are my papers, everything is there what do we have to talk about'?

Even though academic norms and expectations of academe seem clear and selfexplanatory to the researcher, they are not necessarily of any help in the practical setting, and can even lead to a sense of alienation:

[I felt] alienated as a researcher.... A lack of understanding of my professional identity...to put it simple ... I felt like ... like an alien .... UFO. And having landed in this office... it was completely different ... business culture of course, they had never seen a researcher ... it's completely like... technical research and development.

Under these circumstances the participants have to reassess their role as researchers, in terms of what they meant to other organizational members in the capacity of that role position. This would sometimes amount to a delicate social game:

I have been really careful with how I take space and how I talk about things and criticize ... I really paid a lot of attention to building trust in the organization ... so they don't feel that I'm someone who is criticizing what they do.

However, such role conflicts may also have a positive role ambiguity flipside that allows for positively experienced role combinations, e.g.:

I wasn't sure whether I wanted to stay in academia ... but getting a proper job as normal person working for a public agency or as an evaluator ... And I also thought ...that it would be difficult for me to get such a job because as a $\mathrm{PhD}$ in philosophy... you don't hire people with that kind of background... so Flexit obviously was perfect ... because I didn't have to choose ... I could keep both for another few years.

\section{Learning and Development}

While the previous set of quotes illustrate the difficulties involved in participating in the program, the following instead exemplify the various ways in which participants 
experienced learning and development in their host organizations. An important aspect of this relates to culture, especially regarding a positive experience of cooperative work culture vis-à-vis that of academe, e.g.:

... and some people have a background from consulting, management consulting so, that's an interesting mix and we learn a lot from each other... it's team work ...very helpful ... in academia they always fight for who's the author of this, who's the author of that ...here people are very generous.

An unexpected culture clash can be a source of elevation for the researcher, as is clear from the following quote:

It really affected me because, I mean, after years of uncoolness at [university] I was suddenly in this environment ... They didn't understand, but they were ... listening to me ... it was very different.

The above suggests that novelty and uncertainty about circumstances do not necessarily imply negative uncertainty or friction. It can also entail a positive experience. Experiences such as those illustrated in the preceding two quotes are probably crucial if the new cultural experience is to lead to learning and development. It could, for example, involve learning a new language or cultural code:

I'm in business administration so we're quite used to interacting with business ... but I had a hard time using my ... my own kind of language. I couldn't really make myself understood ...I just learned their language instead.

Many of the participants experienced a connection between cultural learning and academic development. This connection came about through various mechanisms, e.g., by developing new relevant competencies and identifying new research problems, and in some cases by developing new research identities. Starting with identity, it is, of course, true that the individuals applying for Flexit were already primed to accept the types of challenges that the program would pose to their research:

I had absolutely no clue whatsoever what it could mean to do research outside of an academic institution... but I was interested in finding new ways of doing research.

However, this being said, their presence in a non-research environment was not necessarily undermining their confidence in the efficacy of their academic personas, quite the opposite. Part of their development as researchers had to do with coming to understand the relative strength of their academic roles in the practical context, e.g.:

I thought they were like hands-on, superefficient all the time, every day (laughs) ... but no it's not true, as a researcher you are quite clear on the methods. So I gained confidence and that's probably something that will come across in my teaching and my way of doing research ...

This also involved coming to understand how specifically academic qualities could bring something novel and important into the new environment: 
They used me...as a researcher but also as an experienced co-worker...... Many people said - it's been so wonderful we can always sit down and reason about things and discuss things, and you're always bringing new contributions and new perspectives to the discussions ... I would say that's to a large extent a combination of my experience and my research role.

In the above case, the respondent experienced a transition to being a more reflective contributor, in his/her role as an academic and practitioner. The researcher's practitioner colleagues strongly supported this transition. Finding a use for one's ability to theorize and reflect in a practical context, represented an important form of learning, but also understanding the limits of a purely academic competence:

My experience here has helped me a lot with ... thinking about ... which of the theoretical concepts and ideas actually work in reality. People stop me and say - now you're becoming too abstract and theoretical and you're losing me ... because I have a different role here they can actually say that to me ...

One might argue that the above quote represents a particular type of experience of competence acquisition, namely, coming to understand how theory and research are useful or not in applied contexts. There were other more hands-on examples of competence development, specifically concerning the research process. These involved learning new ways of doing research. A case in point was one philosopher who was expected to acquire empirical material for the first time:

I would also have a lot of new material to theorize on. Because there is often a lot of theorizing based on no empirics at all... So I could probably theorize on this material for like three years or something. I would write on ... strong empirical material.

Life in a practitioner setting can also offer possibilities to learn academically useful approaches that would benefit not just practice, but also one's own future academic research:

I have had the possibility to learn new methods, for instance group interviews...spent a lot of time studying and learning how to do this and...training ...talking to people who work with these kinds of methods and ...combining that with my background.

While part of the experience of learning and development has to do with methods and practical approaches, there are also a number of ways in which the approach to problems develops. One has to do with the challenge of converting academic to applied knowledge, as in the case where descriptive and theoretical insights must be turned into plans for designing something that actually 'works':

I had a huge amount of research knowledge about... what factors are important for [research object]... This was my research area, so I thought well... now I just have to put my knowledge in [another] perspective... 
Applying existing knowledge implies a shift in what is considered a relevant use for research. However, in the case of the social sciences/humanities, practice also has the potential to offer completely new examples of phenomena that can be explored as legitimate academic inquiry, e.g.,

...it's a project within the sharing economy.... It's new and they don't really know what will happen with it. So I will take the opportunity to do research on it, which I think is really interesting, but also something that can help the organization develop the project into something that is sustainable and efficient and profitable.

In the above qoute, it is clear that the horizons for what is considered a legitimate academic and a useful practical problem have merged for the participant. It may well be that this is the ultimate aim of the Flexit program in terms of desirable effects on participants, since supposedly a merger of academic and practical knowledge development would assume, or at least critically promote, learning and development in participants' role accommodation and cultural breadth as well.

In what follows we will present a synthetic analysis of the above and propose a methodological framework for assessing similar experiences from funding instruments.

\section{Discussion and Conclusions}

We will begin this section by providing an analytical summary of the interview results presented above, with an eye to how they resonate with the cognitive-experiential aspects of the instrument discussed above. The section ends with a few rationales and methodological suggestions for 'experiential impact analysis' for similar types of immersive funding instruments.

As we have seen, one may divide the responses to the Flexit instrument into two broad experiential categories: uncertainty and friction, on the one hand, and learning and development, on the other. The first of these thematizes cognitive/epistemic issues, involving how the new context represents ambiguous expectations, which in turn affect how actors come to understand research problems, and their research agenda. This first category also refers to a social/cultural aspect of uncertainty and friction. We will address these two aspects below starting with the social/cultural dimensions. In the first place, one observes expressions of uncertainty regarding what to do and what is expected from the actor, which involves a normative relationship between the actor and the people around them. What are these expectations founded on, how negotiable are they and how should and could the researcher adapt to them? A disconnect between the actor's and the host's understanding of the context and its challenges leads to uncertainty about the value of one's professional identity and one's research trajectory. Of central import here is the researcher experience as related to a research agenda, with which they can identify. This partly has to do with how one comes to understand the balance between one's interest and that of the organization. This balance seems to some extent to be a matter of ambiguity regarding social identity, e.g., 'when am I a researcher and when am I a colleague?' 
Such balancing may also entail deciding on how to prioritize personal interests visà-vis those perceived to be the organization's interests, even when one's own identity as a researcher is challenged. The tension observed here seemed to be further exacerbated by the fact that the researcher was alone, without anyone to share his/ her experience. We observe the impact on the researcher of an ambiguous experience (or polymorphous to use Kasim and Le Gale's, 2010 expression), in the sense that there are conflicting signals about what to do, and no clear, delineated direction for action. This impact is not, at least not initially, perceived as a positive one by the researcher. In Hood's (1986) terms it is due to a complex and intrusive context, with a low degree of norm-sharing (John 2013) and capacity for action (Lascoumes and Le Gales 2007).

A more social side of uncertainty and friction concerns culture and the role perception of the researcher. Here, the disconnect discussed above is experienced on a 'higher' level, as one between the research culture of academe and the host organization's culture, e.g., in how one delineates and structures problems. Moving from academic clarity about how issues are presented (where methods, theories, and research programs provide a foundation), to a more fluid state, is a matter of cultural as well as cognitive uncertainty. Academic recognition is fairly predictable given certain conditions, however, in the corporate setting it is not, leading to a sense of alienation for the researcher. Ambiguity of role position and professional worth was sometimes connected to a need to play down one's professional identity and impulses, e.g., to criticize and reflect. While this appears to represent a form of professional devaluation, we also observe how sometimes the opposite was true. For example, a person who had previously seen themselves as locked into a narrow academic role now experienced a sort of dual professional identity or extended identity. Instrument impacts on actor experiences of this kind imply a reduced instrumentactor conflict (Doern 1981) and a more voluntary immersive potential of the instrument-world vis-à-vis the actor (Salamon and Elliott 2002). This brings us to the second category, where instrument immersion takes on a more positive character.

In the learning and development aspect of instrument immersion, culture, identity and research process are salient features. Firstly, actors experienced ways of relating in organizational life, different from those typically found in academe, e.g., through new forms of collectivism and mutuality. This was connected to different attitudinal sentiments, for example, by finding a way of interacting that is perceived to be 'cool' and attractive, thus forming a positive counterpoint to previous experiences as a researcher. A recurrent theme was that of challenges and change to research identity. From a learning and development perspective we note how the new setting can stimulate curiosity and open new avenues for attempting inquiry. Also it turns out that it may strengthen a belief in the efficacy of the 'academic persona' across the academic-practitioner divide, for example, when the researcher realizes they possess strength in analyzing and contributing in the practical setting. This includes coming to understand the limits and possibilities of one's academic knowledge/competence in that practical setting. Identity is closely connected to changes in the approach to research that the program brings about. This involves conceptualizing oneself as a new kind of researcher, working with new types of material in new ways, and making different kinds of contributions. By shifting one's view on the 
knowledge one possesses, and by turning it into completely different uses, a form of re-contextualization of knowledge takes place, which is connected to the social re-contextualization the program brings about. The opposite, demonstrated in the interviews, is when a practical problem is re-contextualized into an academic one. Both of these forms of re-contextualization are connected to the complexity and polymorphousness offered up to the participant by the instrument, which affords a freedom to choose, or to use Lascoumes and Le Gale's (2007) phrases, new 'capacities for action and structures of opportunity.' Having touched upon some of the key elements of the findings, we are now able to provide a discussion of what may amount to a framework for analyzing the efficacy of funding instruments from the perspective of their 'experiential impacts.'

\section{Experiential Impact of Funding Instruments: Outline of an Approach}

From our previous discussion, it is clear that an instrument can be experienced as more or less frictious or developmental by the researcher, and that this experience is related to the researcher's goals and identity. Furthermore, it seems, at least from our study, that these goals and identities are mainly cognitive: they relate to research and other types of problem solving. We may ask, following Ihde (1990, 2004), to what extent can an immersive instrument such as Flexit fade into the researcher's background, and be 'seen through' or 'made transparent' in their day-to-day existence? Is the instrument offering a truly immersive rather than a resistant experience? The above raises a second question which is: to what extent does the instrument have the capacity to challenge the researcher, in terms of helping him/her develop in new directions or, for that matter, by creating a barrier to learning? Describing a funding instrument from these perspectives opens up new insights into how to support and develop the instrument, and how to assess its likely outcomes on the researcher. It also allows one to see beyond the dominant orthodoxy of treating funding instruments as mere devices for allocating resources to research. Table 1 is an attempt to locate Flexit in a typology utilizing these dimensions, so that it can be compared to other types of funding instruments.

As illustrated in Table 1, research funding instruments can be understood according to the challenge/immersion dimensions. The location of an instrument in this grid indicates how much the funder can expect the instrument to challenge existing practices and how much auxiliary effort might be needed to guarantee, support or guide the process of immersion. In the case of project funding, neither dimension would make much difference. Center of Excellence funding may be immersive (new environment) but leave research production intact since it would (typically) allow existing trajectories to be pursued. ERC starting grants leave the organizational

Table 1 Challenge and immersion from funding instruments

\begin{tabular}{lll}
\hline & Low immersion & High immersion \\
\hline High challenge & ERC starting grant & Flexit \\
Low challenge & Project funding & Centers of Excellence \\
\hline
\end{tabular}


circumstances more intact (low immersion) while challenging the researcher with a higher degree of autonomy and organizational responsibility than e.g., a project grant would. Our point here is that the more immersive and challenging the instrument, the more analysis is needed to get the instrument mix and implementation process right. In conducting such an analysis a few issues seem especially pertinent given the above. We have already concluded that one may analyze immersive instruments (or any kind of instrument) from the point of view of the experiential challenge it brings about. However, it is also important to note that such challenge, as well as immersion, is multidimensional, as it relates to contextual elements of the instrument-actor world.

Working from the phenomenological conception outlined earlier, we note that an instrument has an immersive potential that is connected to how adapted it is to the actor's characteristics and sentiments (cf. Salamon and Elliott 2002). This has to do with qualities such as adaptability, voluntariness, discernibility, coherence, actor-fit and legitimacy of the instrument. Ihde (1990) describes the quality of such immersion as optimally being one where the instrument fades into the background, because it becomes embodied by the subject. In our case this happens when the researcher has found his/her home (albeit temporarily) in the host organization, and stops experiencing it as difficult. Yet, while the instrument is now transparent to the actor, it is still 'available' in that it reinforces the actor's abilities and goals (Ihde 1990, 2004). In short, the instrument's immersive potential has to do with (1) its ability to fade into the background and become invisible to the actor, and (2) it being non-obvious to the actor, while still being able to constitute an experience of learning and development.

The other dimension, challenge, requires a separate type of analysis. First of all, we recognize that challenges can be experienced as negative or positive (friction/ uncertainty or development/learning). Some negatively experienced challenges are probably necessary for development, but the question is, which are they? Taking our above analysis as point of departure, assessing an instrument from this perspective is about anticipating its legitimacy and fit in terms of its espoused/implied norm structures vis-a-vis the researcher. To what extent one may speak of 'legitimate norm adjustment' as far as challenging the researcher, i.e., asking him/her to change, is concerned (cf. John 2013). This will be both a matter of understanding the norms built into the instrument, and the norms of the actor, as well as the degree of normatively acceptable or 'legitimate' pressure on the actor (Doern 1981).

In conclusion, we suggest that in analyzing the nature, level of challenge and immersion an instrument should project, one needs a normative concept of the actor's identity anchored in his/her goals for knowledge production. Such an 'epistemic identity' would capture ideas about what knowledge to pursue, as well as how, and for what reasons, such ideas come to constitute the boundaries of a researcher's identity. ${ }^{2}$ A key balancing mechanism here is that between epistemic and social norms/factors that trade off, reinforce or undermine mutually in the experience of

\footnotetext{
2 The notions of 'epistemological identity' and 'epistemic identities' are applied in a different sense in Demerath (2006) and Osbeck and Nersessian (2017).
} 
the actor (cf. Rip 1981, 1997). However, we will leave a discussion of the details of this be for the time being. What we hope to have showed in the above is that it is essential for successful funding instrument design to incorporate an understanding of the level of immersion of an instrument, the mechanisms through which it operates on the experience of the actor, and if the challenges it presents are productive or not in researchers' life-world. This being one way among several towards specifying how the researcher experience is central for how instruments affect knowledge production.

Acknowledgments Open access funding provided by Lund University. Funding was provided by RJ, the Swedish Foundation for Humanities and Social Science (Grant No. FSK15-0881:1).

Open Access This article is licensed under a Creative Commons Attribution 4.0 International License, which permits use, sharing, adaptation, distribution and reproduction in any medium or format, as long as you give appropriate credit to the original author(s) and the source, provide a link to the Creative Commons licence, and indicate if changes were made. The images or other third party material in this article are included in the article's Creative Commons licence, unless indicated otherwise in a credit line to the material. If material is not included in the article's Creative Commons licence and your intended use is not permitted by statutory regulation or exceeds the permitted use, you will need to obtain permission directly from the copyright holder. To view a copy of this licence, visit http://creativecommons.org/licen ses/by/4.0/.

\section{References}

Bishop, Pamela, Shuyler W. Huck, Bonny H. Ownley, Jennifer K. Richards, and Gary J. Skolits. 2014. Impacts of an interdisciplinary research center on participant publication and collaboration patterns: A case study of the national Institute for Mathematical and Biological Synthesis. Research Evaluation 23: 327-340.

Brorstad Borlaug, Siri. 2016. Moral hazard and adverse selection in research funding: Centers of Excellence in Norway and Sweden. Science and Public Policy 43(3): 352-362.

Clarysse, Bart, Mike Wright, and Phillippe Mustar. 2009. Behavioural additionality of R\&D subsidies: A learning perspective. Research Policy 38: 1517-1533.

De Rijcke, Sarah, Paul Wouters, Alex D. Rushforth, Thomas P. Franssen, and Björn M.S. Hammarfelt. 2016. Evaluation practices and effects of indicator use-A literature review. Research Evaluation 25(2): 161-169.

Demerath, Loren. 2006. Epistemological identity theory: Reconceptualizing commitment as self-knowledge. Sociological Spectrum 26: 491-517.

Doern, G.B. 1981. The nature of scientific and technological controversy in federal policy formation. Ottawa: Science council of Canada.

Elmore, Richard F. 1987. Instruments and strategy in public policy. Policy Studies Review 7(1): 174-186.

Giorgi, Amadeo, and Barbro Giorgi. 2003. Phenomenology. In Qualitative psychology, ed. J.A. Smith, 25-50. London: Sage Publications.

Gläser, Jochen, and Grit Laudel. 2016. Governing science: How science policy shapes research content. Archives Européennes de Sociologie 57: 117-168.

Gläser, Jochen, and Kathia Serrano Velarde. 2018. Changing funding arrangements and the production of scientific knowledge: Introduction to the special issue. Minerva 56(1): 1-10.

Heidegger, Martin. 1962. Being and time. New York: Harper \& Row.

Hellström, Tomas. 2018. Centres of Excellence and capacity building: From strategy to impact. Science and Public Policy 45(4): 543-552.

Hellström, Tomas, and Merle Jacob. 2017. Policy instrument affordances: A framework for analysis. Policy Studies 38: 604-621.

Hood, Christopher C. 1986. The tools of government. Chatham: Chatham House Publishers. 
Ihde, Don. 1990. Technology and the life-world: From garden to earth. Indiana: Indiana University Press. Ihde, Don. 2004. A phenomenology of technics. In Readings in the philosophy of technology, ed. D.M. Kaplan, 137-159. Lanham, ML: Rowman \& Littlefield.

John, Peter. 2013. All tools are informational now: How information and persuasion define tools. Policy and Politics 41: 605-620.

Kassim, Hussein, and Patrick Le Galés. 2010. Exploring governance in a multi-level polity: A policy instruments approach. West European Politics 33(1): 1-21.

Lascoumes, Pierre, and Patrick Le Galés. 2007. Introduction: Understanding public policy through its instruments-From the nature of instruments to the sociology of public policy instrumentation. Governance 20(1): 1-21.

Laudel, Grit, and Jochen Gläser. 2014. Beyond breakthrough research: Epistemic properties of research and their consequences for research funding. Research Policy 43: 1204-1216.

Linder, Stephen H., and B. Guy Peters. 1989. Instruments of government: Perceptions and contexts. Journal of Public Policy 9(1): 35-38.

Lowi, Theodore J. 1972. Four systems of policy, politics, and choice. Public Administration Review 32(4): 298-310.

Luukkonen, Terttu. 2000. Additionality of EU framework programmes. Research Policy 29(6): 711-724.

Merleau-Ponty, Maurice. 1962. Phenomenology of perception. New York and London: Routledge.

Osbeck, Lisa M., and Nancy J. Nersessian. 2017. Epistemic identities in interdisciplinary science. Perspectives on Science 25: 226-260.

Radaelli, Claudio M., and Anne Meuwese. 2010. Hard questions and equally hard solutions: Proceduralization through impact assessment in the European Union. West European Politics 31(1): 136-153.

Rip, Arie. 1981. A cognitive approach to science policy. Research Policy 10: 294-311.

Rip, Arie. 1997. A cognitive approach to relevance of science. Social Science Information 36(4): 615-640.

RJ [Swedish Foundation for Social Science and the Humanities]. 2018. https://www.rj.se/en/applying-forgrants/Flexit/. Accessed September 10, 2018

Salamon, Lester M. 1981. Rethinking public management: Third-party government and the changing forms of government action. Public Policy 29(3): 256-275.

Salamon, Lester M., and Odus V. Elliott. 2002. The tools of government: A guide to the new governance. Oxford: Oxford University Press.

Smith, Jonathan A. 1996. Beyond the divide between cognition and discourse: Using interpretative phenomenological analysis in health psychology. Psychology and Health 11: 261-271.

Smith, Jonathan A., and Mike Osborn. 2003. Interpretative phenomenological analysis. In Qualitative Psychology, ed. J.A. Smith, 51-80. London: Sage Publications.

Weibel, David, Bartholomäus Wissmath, and Fred W. Mast. 2010. Immersion in mediated environments: The role of personality traits. Cyberpsychology, Behavior, and Social Networking 13: 251-256.

Whitley, Richard. 2003. Competition and pluralism in the public sciences: The impact of institutional frameworks on the organisation of academic science. Research Policy 32: 1015-1029.

Whitley, Richard. 2007. Changing governance of the public sciences: The consequences of establishing research evaluation systems for knowledge production in different countries and scientific fields. In The changing governance of the sciences, eds. R. Whitley and J. Gläser, 3-27. Dordrecht: Springer Publications.

Witmer, Bob G., and Michael J. Singer. 1998. Measuring presence in virtual environments: A presence questionnaire. Presence: Teleoperators and Virtual Environments 7: 225-240.

Publisher's Note Springer Nature remains neutral with regard to jurisdictional claims in published maps and institutional affiliations. 